EPJ Web of Conferences 113,03002 (2016)

DOI: $10.1051 /$ epjconf/201611303002

CC Owned by the authors, published by EDP Sciences, 2016

\title{
Resonance model for the three-body states of the $A=6$ reactions
}

\author{
Mark W. Paris ${ }^{1, a}$ and Gerald M. Hale ${ }^{1, b}$ \\ ${ }^{1}$ Theoretical Division (T-2), Los Alamos National Laboratory, Los Alamos NM 87545, USA
}

\begin{abstract}
We present an $R$-matrix-based model for three-body final states that has aspects of the Faddeev approach to three-particle scattering. The model is applied to describing the nucleon spectra for breakup reactions in the $A=6$ systems. Calculations using a charge-symmetric parametrization agree fairly well with the experimental data, although they indicate larger contributions from the ${ }^{5} \mathrm{He}$ or ${ }^{5} \mathrm{Li}$ ground state are necessary.
\end{abstract}

\section{Introduction}

There has been much interest and experimental activity lately at ICF facilities, such as OMEGA at the U. of Rochester, and the NIF at Livermore, to measure the particle spectra from three-body breakup reactions initiated by the laser implosion of capsules containing tritium $\left(t={ }^{3} \mathrm{H}\right),{ }^{3} \mathrm{He}$, or a mixture of the two. Here we describe an $R$-matrix-based model for fitting these spectra that maintains some aspects of the Faddeev method for three-particle scattering. The next section gives a brief introduction to the concepts of $R$-matrix theory and summarizes the resonance model, in which the $T$ matrix is expressed as the sum of three Faddeev-like components. When specialized to the case of single-level terms, these expressions form the basis of our present model, which is similar to that of Brune et al. [1], but differs from it in detail.

The application of these formulas to calculate the spectra of nucleons from the 3-body breakup of the reactions induced by $t+t,{ }^{3} \mathrm{He}+{ }^{3} \mathrm{He}$, and $t+{ }^{3} \mathrm{He}$ is described in Section 3. These calculations use charge-symmetric (CS) parameters for the quantities dominated by nuclear forces, but take into account the Coulomb differences in the resonant sub-systems. This CS approach clearly has more justification for the first two (pure $T=1$ ) mirror reactions than it does for the third, which is an isospin mixture of $T=1$ and $T=0$. The conclusions from this study thus far and the outlook for future work are given in Section 4.

\section{R-matrix based resonance model}

$R$-matrix theory asserts that, due to the short range of nuclear forces, there exists a finite region of coordinate space beyond which the scattering wave function for an $A$-nucleon system assumes its known asymptotic form in terms of $T$-matrix amplitudes. Through the device of the Bloch operator,

\footnotetext{
a e-mail: mparis@lanl.gov

be-mail: ghale@lanl.gov
} 
$\mathcal{L}_{B}$, acting at the surface enclosing that region, a complete set of eigenfunctions of the operator $H+\mathcal{L}_{B}$ can be used to expand the scattering wave function inside it as

$$
\begin{gathered}
\phi_{\mathbf{q}}^{+}(\mathbf{x})=\sum_{\lambda} c_{\lambda}(\mathbf{q}) \phi_{\lambda}(\mathbf{x}), \\
c_{\lambda}(\mathbf{q})=\frac{1}{\sqrt{\pi}} \sum_{\lambda^{\prime}} A_{\lambda \lambda^{\prime}} \gamma_{\lambda^{\prime}} O^{-1}(q) \mathcal{Y}_{s l}^{j \pi}(\hat{\mathbf{q}}) .
\end{gathered}
$$

In Eq. (2), $A_{\lambda \lambda^{\prime}}$ is the level matrix, $\gamma_{\lambda^{\prime}}$ is the reduced-width amplitude, $O$ is the outgoing-wave solution, and $\mathcal{Y}_{s l}^{j \pi}$ is the spin-angle eigenfunction of total angular momentum and parity.

In the resonance model, a two-body initial state reacts to give a three-body final state. The assumed Jacobi coordinates and potentials in the final state are shown in Fig. 1. The pair-wise interactions in the final state are treated as in the Faddeev approach to three-particle scattering, but in these applications to the $A=6$ systems, the initial state cannot be formed from a bound state of any pair of particles in the final state, as is required by the Faddeev theory. Hence, we call it "Faddeev-like."

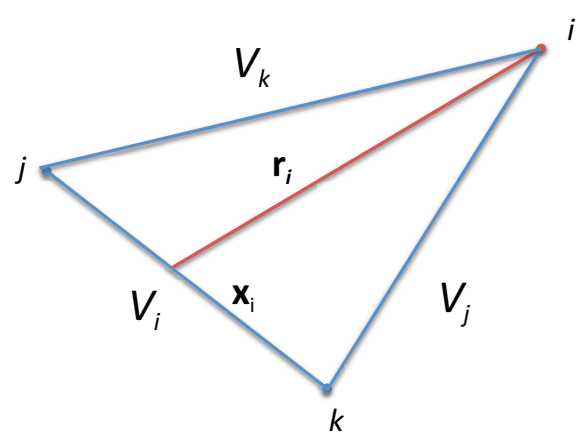

Figure 1: Jacobi coordinates and pair-wise potentials for describing a three-body final state.

The three-body breakup transition amplitude can be written as a sum of Faddeev-like components

$$
\begin{gathered}
T^{(3)}=\sum_{i=1}^{3} T_{\mathbf{q}_{\mathbf{i}} \mathbf{k}_{\mathbf{i}} \mathbf{k}_{\mathbf{0}}}^{(3)}, \\
T_{\mathbf{q}_{\mathbf{i}} \mathbf{k}_{\mathbf{i}} \mathbf{k}_{\mathbf{0}}}^{(3)}=-\pi\left\langle\chi_{\mathbf{q}_{i}} \chi_{\mathbf{k}_{i}}\left|V_{i}\right| \psi_{\mathbf{k}_{0}}^{+}\right\rangle,
\end{gathered}
$$

where $\psi_{\mathbf{k}_{0}}^{+}$is the scattering solution in the initial two-body state, and $\chi_{\mathbf{q}_{i}}$ and $\chi_{\mathbf{k}_{i}}$ are the plane-wave solutions for the momenta $\mathbf{q}_{i}$ and $\mathbf{k}_{i}$ conjugate to $\mathbf{x}_{i}$ and $\mathbf{r}_{i}$, respectively. Relating the plane-wave solution $\chi_{\mathbf{q}_{i}}$ on the left side of the matrix element to the two-body scattering solution $\phi_{\mathbf{q}_{i}}^{-}$through the Lippmann-Schwinger equation gives Eq. (4) as the distorted-wave $T$-matrix element

$$
T_{\mathbf{q}_{\mathbf{i}} \mathbf{k}_{\mathbf{i}} \mathbf{k}_{\mathbf{0}}}^{(3)}=-\pi\left\langle\phi_{\mathbf{q}_{i}}^{-} \chi_{\mathbf{k}_{i}}\left|V_{i}+V_{i} G_{0 i}^{+} V_{i}\right| \psi_{\mathbf{k}_{0}}^{+}\right\rangle .
$$

The $R$-matrix phenomenology is introduced by making in each of the Jacobi arrangements the expansion of Eqs. (1) and (2) for $\phi_{\mathbf{q}_{i}}^{-}$, giving for the 3-body breakup $T$ matrix,

$$
T^{(3)}=\sum_{i=1}^{3} \sum_{\lambda_{i}} c_{\lambda_{i}}^{(i)}\left(\mathbf{q}_{i}\right) \tilde{T}_{\lambda_{i} \mathbf{k}_{i} \mathbf{k}_{0}}^{(2)},
$$


with

$$
\begin{aligned}
\tilde{T}_{\lambda_{i} \mathbf{k}_{i} \mathbf{k}_{0}}^{(2)} & =-\pi\left\langle\phi_{\lambda_{i}}^{(i)} \chi_{\mathbf{k}_{i}}\left|V_{i}+V_{i} G_{0 i}^{+} V_{i}\right| \psi_{\mathbf{k}_{0}}^{+}\right\rangle \\
& =\sum_{J s^{\prime} l^{\prime} s l} \boldsymbol{Y}_{\lambda J s^{\prime} l^{\prime}}(\hat{\mathbf{k}}) O_{l^{\prime}}^{-1}(k) R_{\lambda s^{\prime} l^{\prime} s l}^{\bar{L} l}(\varepsilon) O_{l}^{-1}\left(k_{0}\right) \mathcal{Y}_{J s l}^{*}\left(\hat{\mathbf{k}}_{0}\right),
\end{aligned}
$$

a pseudo two-body off-energy-shell $T$-matrix element expressed in terms of the outgoing-wave $R$ matrix amplitudes $R_{\lambda s^{\prime} l^{\prime} s l}^{\bar{L}}(\varepsilon)$. In the applications described below, all the expansions are single-level $\left(\lambda=\lambda^{\prime}=1\right)$, the resonance parameters in the $c_{\lambda}$ are determined by outside data, and only the magnitudes of the $R$-matrix amplitudes are adjusted to fit the experimental spectra.

\section{Applications to $A=6$ spectra}

The single-level resonance parameters for the ground and first-excited states of ${ }^{5} \mathrm{He}$ and ${ }^{5} \mathrm{Li}$ were taken from analyses of $n+\alpha$ and $p+\alpha$ scattering. Similarly, those for the singlet $n-n$ and $p-p$ resonances were taken from a charge-independent analysis of $N+N$ scattering. The $R$-matrix amplitudes were first adjusted by hand to fit the $t+t$ spectra of Wong et al. [2], as shown in Fig. 2. Then those same amplitudes were used to predict the proton spectrum for the ${ }^{3} \mathrm{He}+{ }^{3} \mathrm{He}$ reaction shown in Fig. 3, and for the $t+{ }^{3} \mathrm{He}$ reaction shown in Fig. 4 .

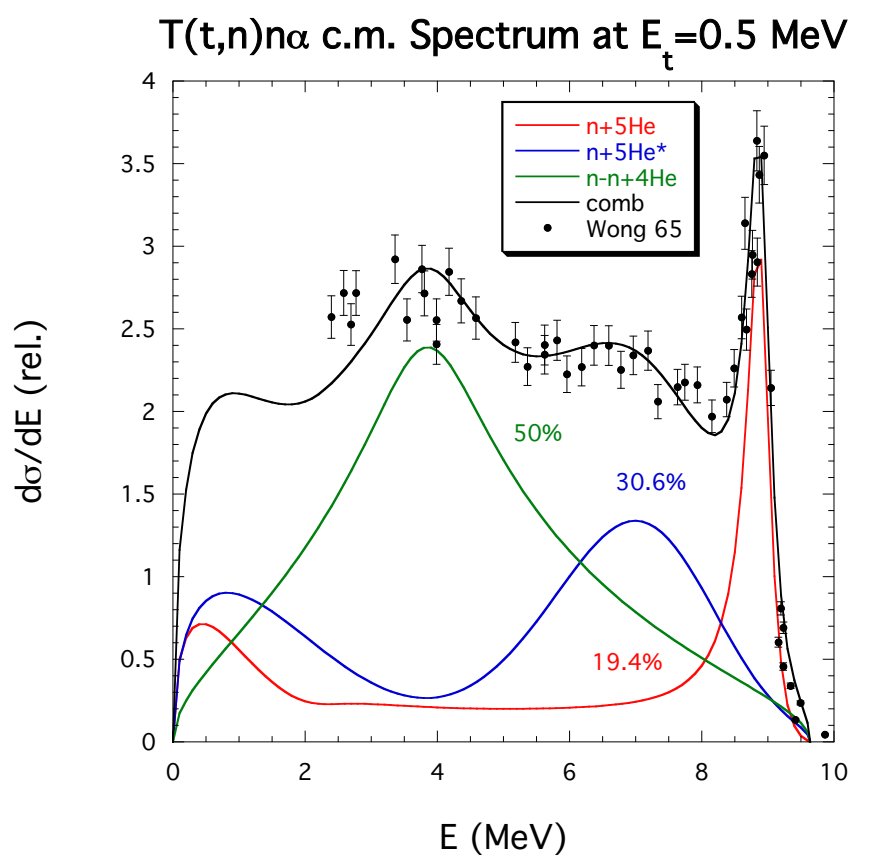

Figure 2: Neutron spectrum from the $t+t$ reaction at $E_{t}=0.5 \mathrm{MeV}$. The data are from Ref. [2], and the colored lines give the 3 Jacobi components of the spectrum with their branching ratios. 

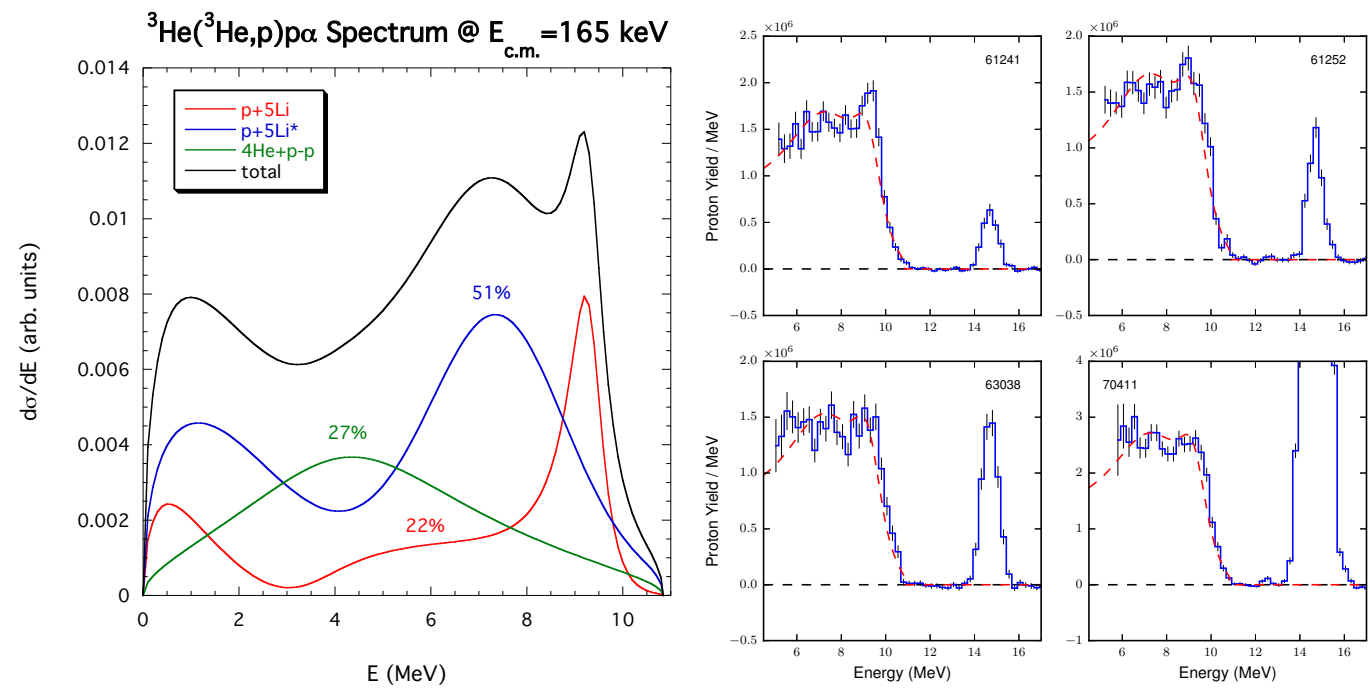

Figure 3: Proton spectra from the ${ }^{3} \mathrm{He}+{ }^{3} \mathrm{He}$ reaction at $E_{c . m} .=165 \mathrm{keV}$. The left side of the figure shows the unbroadened spectrum with its 3 components, and the right side shows the instrumentally broadened spectrum compared with data from four different OMEGA shots [3, 4].
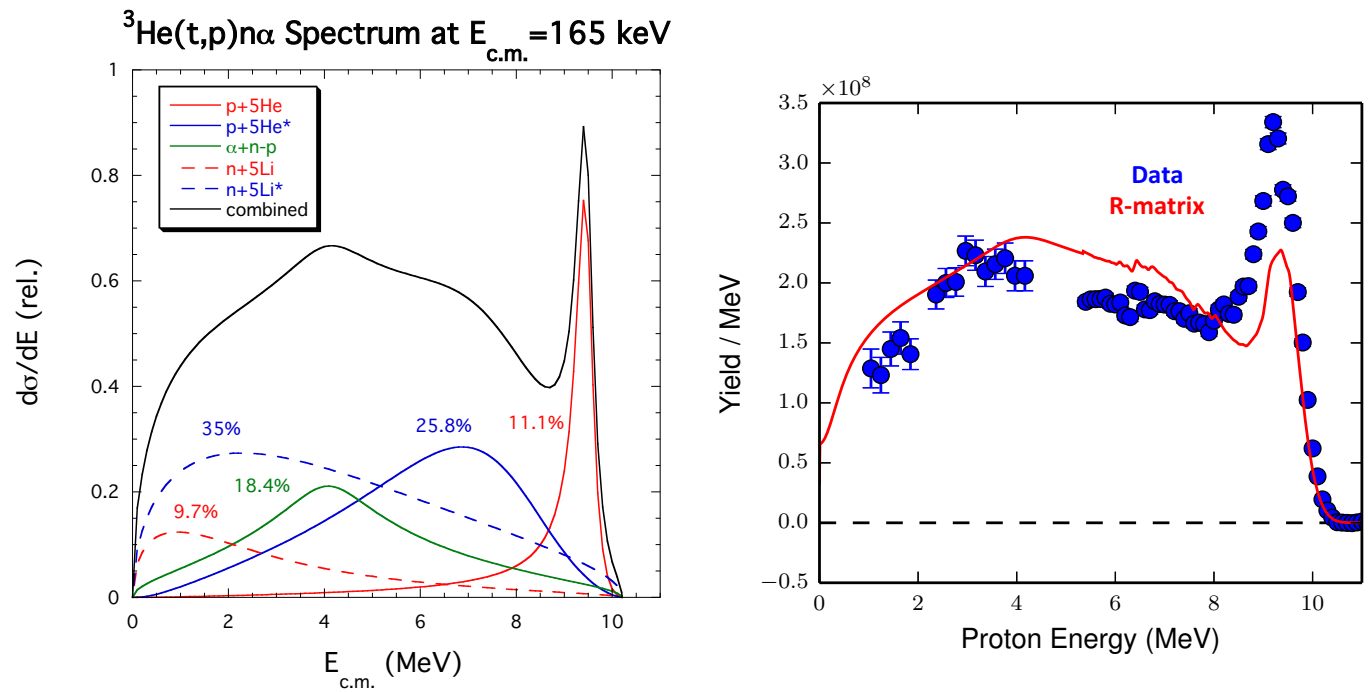

Figure 4: Proton spectra from the $t+{ }^{3} \mathrm{He}$ reaction at $E_{c . m} .=165 \mathrm{keV}$. The left side of the figure shows the unbroadened spectrum with its 5 components, and the right side shows the instrumentally broadened spectrum compared with OMEGA data [3, 4]. 


\section{Conclusions and outlook}

The agreement with experimental $A=6$ spectra is reasonable. Coulomb differences in the resonances are important, and lead to different branching ratios for the components of the spectra even with CS amplitudes. However, the peaks at the ends of the spectra coming from the ground states of ${ }^{5} \mathrm{He}$ and ${ }^{5} \mathrm{Li}$ are under-predicted, and the details of the interference terms could be improved. Future plans are to make the fitting automated, include the angular distribution and interference effects with fewer approximations, make the kinematics relativistic, and to determine the energy dependence of the $R^{\bar{L}}$ amplitudes by linking them to $R$-matrix calculations of the integrated break-up cross sections.

\section{Acknowledgements}

We are indebted to our colleagues Andrew Bacher, Carl Brune, Johan Frenje, and Alex Zylstra for valuable discussions about theory and ICF experiments, and for folding our calculations with instrumental response functions in order to compare with their experimental data. This work was supported under the auspices of the US Department of Energy.

\section{References}

[1] C.R. Brune et al., Phys. Rev. C 92014003 (2015)

[2] C. Wong, J.D. Anderson and J.W. McClure, Nucl. Phys. 71, 106-112 (1965)

[3] A. B. Zylstra et al., to be submitted to Phys. Rev. Lett. (2015)

[4] J.A. Frenje et al., plenary talk, this conference (2015) 